\title{
A Case Report of Fractured Tibia with Improved Outcomes through Early Physiotherapy Approach
}

\author{
Anushree Pawar', Heena Pathan', Ruchita Rao', Gaurav Mishra² \\ ${ }^{1}$ Ravi Nair Physiotherapy College, Professor Department of Radiodiagnosis, Jawaharlal Nehru \\ Medical College, Datta Meghe Institute of Medical Sciences, Sawangi (M), Wardha \\ ${ }^{2}$ Department of Radiodiagnosis, Jawaharlal Nehru Medical College, Datta Meghe Institute \\ of Medical Sciences, Sawangi (M), Wardha \\ Corresponding author email: anu.pawar.amp@gmail.com
}

\section{ABSTRACT}

Fracture of proximal one-third of tibia constitute 5-12\% of all tibial fractures. Fractures at the proximal tibia are extensions of fracture into the proximal metaphyseal region of the bone. Treatment of this kind of fractures poses a lot of challenges and depend upon the severity of injury. Treatment options include mainly fixation with open plating. This report reflects on the physiotherapy approach and protocol to augment early recovery and restoring the normal functioning. A 51 year old female reported with complaint of pain and difficulty in performing knee movement as well as unable to bear weight on the affected extremity. She presented history of trauma to right leg in bike accident after which is started experiencing pain, swelling and inability to bear weight on her right leg. She was diagnosed with proximal one-third tibial fracture of right leg for which she has undergone open reduction internal fixation with plate osteosynthesis for fractured tibia. Fracture of proximal one-third right tibia. Therapeutic intervention included range of motion exercises, therapeutic modalities, strengthening exercises and gait training. Final outcome was restoration of the normal function resuming daily activities in a smooth manner. Early physiotherapy intervention followed by fracture fixation helped in functional restoration early recovery.

\section{KEY WORDS: PROXIMAL TIBIA FRACTURE, FIXATION, PHYSIOTHERAPY.}

\section{INTRODUCTION}

The proximal tibia is roughly triangular in cross sectional area and surface proximal to it is almost completely articular, preventing usage of nail entry, which is collinear with the medullary canal. The metaphysis of tibia comprises thin cortical shell which is filled with cancellous bone. Fractures at the proximal tibia are mainly considered to those with extension into the proximal metaphyseal region of the bone. These fractures can be intra-articular or extra-articular in nature. Fractures can occur from

Biosc Biotech Res Comm P-ISSN: 0974-6455 E-ISSN: 2321-4007

\section{rossef}

Identifiers and Pagination

Year: 2021 Vol: 14 No (6) Special Issue

Pages: 57-61

DOI: $h t t p: / / d x . d o i . o r g / 10.21786 / b b r c / 14.6 .14$
This is an open access article under Creative

Commons License Attribn 4.0 Intl (CC-BY). low energy mechanism like athletic injuries, fall from standing height, but they are more often associated with high-energy traumatic mechanisms. Difficulties arise while treating these fractures because of various unique characteristics of proximal leg. Above all, a small sized proximal fragment at times makes reduction control more challenging, regardless of whatever treatment is being chosen. In addition to this, forces acting on the proximal tibia might land up into malalignment. Soft tissue attachments including collateral and cruciate ligaments injury may occur in conjunction with fracture of tibia and can further cause knee instability (James et al., 2003).

Minimally invasive plate osteosynthesis has become widely used technique. The plating done acts as an internal fixator in the bridging manner, thus results in the process of secondary bone healing (Hasenboehler et al., 2006). This minimal invasive technique ensures good outcome

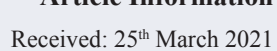

ccepted after revision: $24^{\text {th }}$ May 2021 
in the treatment of proximal tibial fracture (Dankai, et al., 2015). Surgical stabilization is an improved method for the treatment of fractures in the proximal tibia and physiotherapy is an integral aspect for recovery of patients post-surgical management. Pain, effusion and reduced exercise tolerance forms serious issue for the patients postoperatively. But precise protocol used, helps to correct joint range of motion, restore the knee function and thus progress towards the recovery (Tasheva et al., 2020). Early range of motion exercises with early weight bearing needs to be encouraged since it promotes better rehabilitative outcomes (EfthymiosIliopoulas, 2020).

Patient Information: A 51 year old female residence of Samudrapur visited orthopedics department who referred her for physiotherapy came with complained of pain and difficulty in performing knee movement as well as unable to bear weight on the affected extremity. She presented history of travelling through bike when there was collision with another bike sustaining injury to her right leg after which is started experiencing pain, swelling and inability to bear weight on her right leg. Immediately she was taken to orthopedic surgeon investigations were performed and was diagnosed with proximal one-third tibial fracture of right leg for which she has undergone open reduction internal fixation with plate osteosynthesis for fractured tibia.

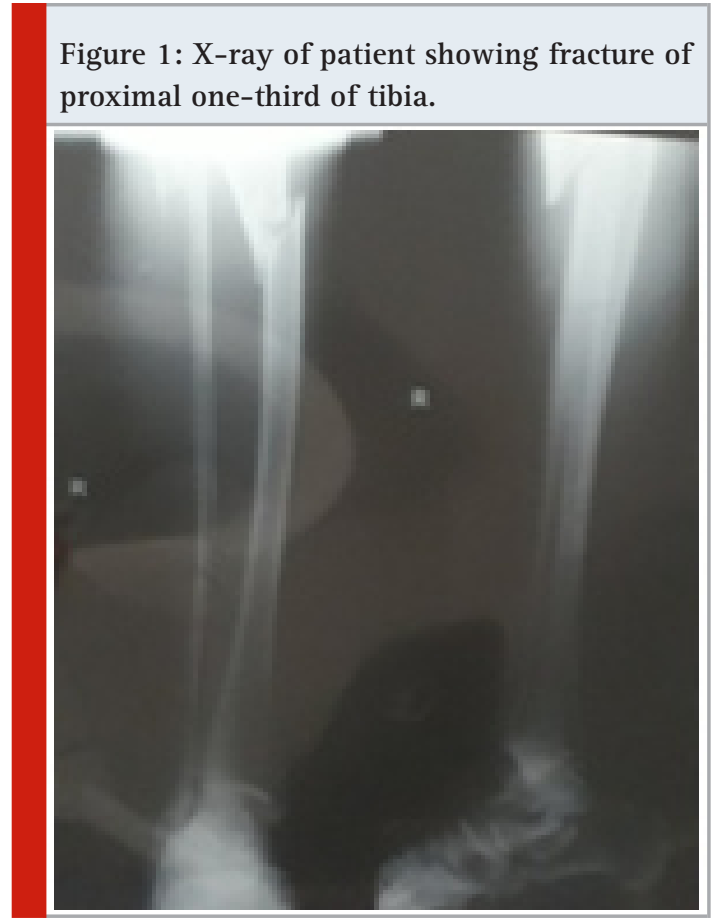

Post operatively patient was treated with analgesics and antibiotics. Later brace was applied for about 6 weeks over the right leg and physiotherapy was started from the day one after fixation and application of brace. Post-operatively patient had complained of pain over the anterior aspect of tibia and on performing movement of the knee joint, it was 8/10 on numerical pain rating scale. Also there was reduction in range of motion, inability to bear weight over the extremity and difficulty in performing lower limb activities such as the activities of daily living.

Clinical Findings: After taking proper informed consent patient was examined in lying position. Affected lower limb in full extension properly supported on pillows and kept elevated. Scar mark was present on the anterior aspect of leg extending from just below the tibial tubercle to mid shaft region. On palpation, local temperature was not raised. Tenderness was present with grade 2 . On neurologic assessment it was found to be normal.

\section{Timeline}

Table 1. Range of motion of joints; pre-treatment assessment.

\begin{tabular}{|l|c|}
\hline Date of injury & $09 / 01 / 2019$ \\
\hline Visit to orthopaedician & $09 / 01 / 2019$ \\
\hline Date of operation & $11 / 01 / 2019$ \\
\hline Physiotherapy rehabilitation & $12 / 01 / 2019$ \\
\hline
\end{tabular}

Therapeutic Intervention: Patient had undergone physiotherapy session for the duration of 12 weeks regularly for all seven days in a week. This session was started with a goal to restore range of motion, strength, improve flexibility and hence facilitate early recovery of the patient.

Table 2. Manual muscle testing; pre-treatment assessment.

\begin{tabular}{|l|c|c|c|}
\hline \multirow{3}{*}{ Joint } & Movements & Active & Passive \\
\hline \multirow{3}{*}{ Hip } & Flexion & 200 & 300 \\
\cline { 2 - 4 } & Extension & 100 & 150 \\
\cline { 2 - 4 } & Abduction & 300 & 350 \\
\cline { 2 - 4 } & Adduction & 300 & 350 \\
\cline { 2 - 4 } & External rotation & 100 & 150 \\
\cline { 2 - 4 } & Internal rotation & 100 & 120 \\
\hline \multirow{4}{*}{ Knee } & Flexion & 00 & 00 \\
\cline { 2 - 4 } & Extension & 00 & 00 \\
\hline \multirow{3}{*}{ Ankle } & Dorsiflexion & 50 & 70 \\
\cline { 2 - 4 } & Plantar flexion & 100 & 150 \\
\cline { 2 - 4 } & Inversion & 100 & 150 \\
\cline { 2 - 4 } & Eversion & 50 & 70 \\
\hline
\end{tabular}

\begin{tabular}{|l|c|c|}
\multirow{3}{*}{ Knee } & Flexors & 0 \\
\cline { 2 - 3 } & Extensors & 0 \\
\hline \multirow{2}{*}{ Ankle } & Dorsiflexors & $1+$ \\
\cline { 2 - 3 } & Plantar flexors & $2+$ \\
\hline
\end{tabular}

0-4 WEEKS: Goal of physiotherapy interventions during this early phase was to reduce pain, swelling and then gently initiate an activity. Physiotherapy was started early even when brace was on beginning from day one in the state of immobilization. To reduce pain, cryotherapy 
was implicated, which was applied for about 10 minutes and to reduce swelling leg was kept in elevation to augment adequate circulation. Patient was encouraged to perform toe movements of affected leg, ten repetition for several times in a day. Strengthening using static quads and range of motion exercises for hip, knee, and ankle were begun soon for an unaffected leg to maintain its mobility.

\begin{tabular}{|c|c|c|c|c|c|}
\hline \multirow[b]{2}{*}{ Joint } & \multirow[b]{2}{*}{ Movements } & \multicolumn{2}{|c|}{ Pre-assessment } & \multicolumn{2}{|c|}{ Post-assessment } \\
\hline & & Active & Passive & Active & Passive \\
\hline \multirow[t]{6}{*}{ Hip } & Flexion & $20^{\circ}$ & $30^{\circ}$ & $110^{\circ}$ & $120^{\circ}$ \\
\hline & Extension & $10^{\circ}$ & $15^{0}$ & $15^{0}$ & $20^{\circ}$ \\
\hline & Abduction & $30^{\circ}$ & $35^{0}$ & $40^{\circ}$ & $45^{\circ}$ \\
\hline & Adduction & $30^{\circ}$ & $35^{0}$ & $40^{\circ}$ & $45^{\circ}$ \\
\hline & External rotation & $10^{\circ}$ & $15^{0}$ & $40^{\circ}$ & $45^{\circ}$ \\
\hline & Internal rotation & $10^{0}$ & $12^{0}$ & $35^{\circ}$ & $40^{\circ}$ \\
\hline \multirow[t]{2}{*}{ Knee } & Flexion & $0^{0}$ & $0^{0}$ & $110^{\circ}$ & $120^{\circ}$ \\
\hline & Extension & $0^{\circ}$ & $0^{0}$ & $0^{0}$ & $5^{0}$ \\
\hline \multirow[t]{4}{*}{ Ankle } & Dorsiflexion & $5^{0}$ & $7^{0}$ & $10^{\circ}$ & $20^{\circ}$ \\
\hline & Plantar flexion & $10^{\circ}$ & $15^{0}$ & $20^{\circ}$ & $45^{\circ}$ \\
\hline & Inversion & $10^{\circ}$ & $15^{0}$ & $25^{0}$ & $35^{\circ}$ \\
\hline & Eversion & $5^{0}$ & $7^{0}$ & $10^{\circ}$ & $10^{\circ}$ \\
\hline
\end{tabular}

Table 4. Strength of muscles of lower extremity pre and post treatment assessment.

\begin{tabular}{|l|c|c|c|}
\hline \multirow{2}{*}{ Knee } & $\begin{array}{c}\text { Pre } \\
\text { treatment }\end{array}$ & $\begin{array}{c}\text { Post } \\
\text { treatment }\end{array}$ \\
\hline \multirow{2}{*}{ Ankle } & Flexors & 0 & $4+$ \\
\cline { 2 - 4 } & Extensors & 0 & $4+$ \\
\cline { 2 - 4 } & Dorsiflexors & $2+$ & 4 \\
\hline
\end{tabular}

5-8 Weeks: Swelling subsided at this time however, there was presence of pain which was managed via application of hydrocollator pack for 10-15minute. It had helped to induce relaxation and reduce pain. In this phase we mainly focused on improving range of motion of ankle, knee along with improvising flexibility and most crucial, the strengthening of muscles of lower extremity of operated side. Range of motion exercise incorporated heel slides, hip flexion, abduction, ankle plantarflexion and dorsiflexion movement of an affected extremity, beginning from the passive movement till the patients tolerance. Strengthening exercises; static hamstrings, quadriceps, calf's were taught to the patient so as to overcome the muscle weakness caused by fracture in the affected extremity. Range of motion exercises along with strengthening protocol which was begun during early 4 weeks was continued for the unaffected leg to maintain its mobility.

9-12 Weeks: All the range of motion exercise as well as the strengthening exercise given in the initial phases were continued and progressed. Passive movements were replaced by active assisted movement. After nine weeks we started with partial weight bearing exercises using crutches under our supervision and timely correction of gait pattern. Gait re-education formed an important component of this stage. Patient was gradually trained with the phases of gait cycle and later on progressed towards weight bearing. Balance training plus proprioception were further inculcated in this treatment protocol.

Follow up and outcome: Table 3 shows pre and post treatment values for range of motion and manual muscle testing. In this phase of 12 weeks patient's condition had shown linear growth in improvement and early recovery was reported.

\section{DISCUSSION}

In this case, patient presented with the complaints of pain, swelling, reduced range of motion and strength in right lower extremity. After clinical evaluation a plan of care was made which included range of motion exercise, strength training, use of therapeutic modalities and gait re-education. There is paucity of articles based upon the important role of rehabilitation protocol following this proximal tibial fracture. Surprisingly, in the literature there is paucity of information regarding the rehabilitation of such patients. Hence, my study would be extensively helpful while treating patients with proximal tibial fracture. Physical therapy is vital part of the patient's rehabilitation in their journey of returning back to pre-injury activity state or at least nearer to that state. It can help in preventing certain problems or helps to focus on the areas that patients need to gain better outcomes. Early range of motion exercises with early weight bearing has to be encouraged (James, et al., 2020; James, et al., 2017). 
Precise physiotherapy helps to correct substitution movements and progress the recovery of patient after surgical stabilization of fractured tibia (Murray et al., 2020; Murray et al., 2020). Early active knee range of motion but limiting weight bearing upto six to eight weeks is recommended. Novel protocols include; pain management, improve range of motion, early weight bearing that facilitate rehabilitation process and improve patients outcome (Vos, et al., 2019). Few of the studies related to fractures of long bones were reported (Dhankar, 2019; Reddy et al., 2019; Dongre, et al., 2019; Gaidhane et al., 2018). Nikose et. al. reported o impact of medial openwedge high tibial osteotomy for medial compartment osteoarthritis of the knee. (Samal et. al. 2010) reflected on minimal invasive percutaneous plate osteosynthesis for distal tibial fracture. Other related articles were reviewed (Bawiskar et al, 2020., Phansopkar et al., 2020).

\section{CONCLUSION}

The above study concludes that definitive surgical approach with early physiotherapy rehabilitation enhanced the functional outcome of patient and it has given a comprehensive recovery plan that helped her in relieving pain, regaining range of motion, strength and could bear weight on her affected extremity post injury. Patient was able to conduct her daily activity in quite better way.

Authors contribution: All authors made best contribution for the concept, assessment, data acquisition, analysis and the interpretation of data.

\section{Conflict of interest: None}

\section{Funding support: Nill}

Patient consent: Proper consent was taken from patient for writing this case report.

\section{REFERENCES}

Bawiskar, Dushyant, Sapna Dhote, and Pratik Phansopkar. 2020 Early Physical Rehabilitation PostSurgery in a Complex Type 5 Schatzker Tibial Plateau Fracture Improves Functional Outcomes: A Case Report. MEDICAL SCIENCE 24: 2675-82.

Dankai Wu, GuangkaiReng, Ankit Shrivastava, Ying Yu, Yueyang Zhang, Chuangang Peng. 2015 International Journal of Clinical and Experimental Medicine. 8(8):13455-13463.

Dhankar, S., and A. Bele. 2019 A Case of Fracture Shaft Femur in a Patient with Transtibial Amputation. Journal of Datta Meghe Institute of Medical Sciences University 14(4): 394-96. https://doi.org/10.4103/ jdmimsu.jdmimsu_190_19.

Dongre, P., A.V. Chandak, A. Singam, and A.V. Bapat. 2019 Evaluation of Low Dose Dexmedetomidine and Neostigmine with Bupivacaine for Post-Operative Epidural Analgesia in Lower Limb Orthopedic Surgeries. International Journal of Pharmaceutical
Research 11: 1874-77. https://doi.org/10.31838/ ijpr/2019.11.02.216.

E. Hasenboehler, D. Rikli, R.Babst. 2006, Locking compression plate with minimally invasive plate osteosynthesis in diaphyseal and distal tibial fracture: A retrospective study of 32 patients. Injury, International Journal Care Injured. 38:365-370

EfthymiosIliopoulas, Nikiforos Galanis. 2020, Physiotherapy after tibial plateau fracture fixation. A systematic review of the literature. 8:1-6.

Gaidhane A, Sinha A, Khatib M, Simkhada P, Behere P, Saxena D, et al. 2018 A systematic review on effect of electronic media on diet, exercise, and sexual activity among adolescents. Indian Journal of Community Medicine. 43(5):S56-65. https://doi.org/10.4103/ijcm. IJCM_143_18.

James C. Krieg.2003, Proximal tibial fractures: current treatment, results and problems. Injury, International Journal Care Injured. 34(1):A2-10.

James, Spencer L, Chris D Castle, Zachary V Dingels, Jack T Fox, Erin B Hamilton, Zichen Liu, Nicholas L S Roberts, et al. 2017 Global Injury Morbidity and Mortality from 1990 to 2017: Results from the Global Burden of Disease Study 2017. Injury Prevention 26(1): i96-114. https://doi.org/10.1136/injuryprev-2019043494.

James, Spencer L, Chris D Castle, Zachary V Dingels, Jack T Fox, Erin B Hamilton, Zichen Liu, Nicholas L S Roberts, et al. 2017, Estimating Global Injuries Morbidity and Mortality: Methods and Data Used in the Global Burden of Disease 2017 Study. Injury Prevention 26(1); i125-53. https://doi.org/10.1136/injuryprev2019-043531.

Latchoumi, T.P., Ezhilarasi, T.P. and Balamurugan, K., 2019. Bio-inspired weighed quantum particle swarm optimization and smooth support vector machine ensembles for identification of abnormalities in medical data. SN Applied Sciences, 1(10), pp.1-10.

Mundada, G., S. Khan, S. Singhania, V. Gupta, P. Singh, and S. Khan. 2017 Type-I Monteggia with Ipsilateral Fracture of Distal Radius Epiphyseal Injury: A Rare Case Report. Annals of African Medicine, 16 30-32. https:// doi.org/10.4103/aam.aam_55_16.

Murray, Christopher J L, Aleksandr Y Aravkin, Peng Zheng, Cristiana Abbafati, Kaja M Abbas, Mohsen Abbasi-Kangevari, Foad Abd-Allah, et al. 2020 Global Burden of 87 Risk Factors in 204 Countries and Territories, 1990-2019: A Systematic Analysis for the Global Burden of Disease Study. The Lancet 396:122349. https://doi.org/10.1016/S0140-6736(20)30752-2.

Murray, Christopher J L, Cristiana Abbafati, Kaja M Abbas, Mohammad Abbasi, Mohsen Abbasi-Kangevari, Foad Abd-Allah, Mohammad Abdollahi, et al. 2019 Five Insights from the Global Burden of Disease Study. The Lancet 396:1135-59. https://doi.org/10.1016/S01406736(20)31404-5.

Nikose, Sunil Sheshrao, Devashree Nikose, Aditya L. 
Kekatpure, Shashank Jain, Kiran Saoji, and Sridhar M. Reddy. 2020 Impact of Medial Open-Wedge High Tibial Osteotomy for Medial Compartment Osteoarthritis of the Knee. WORLD JOURNAL OF ORTHOPEDICS. https://doi. org/10.5312/wjo.v11.i12.606.

Phansopkar, Pratik, Priya Tikhile, Radhika Sawal, Prasad Dhage, Devyani Purushe, and Waqar M. Naqvi. 2020 Early Physiotherapy Rehabilitation Approach Enhances Recovery in Rare Acute Tibial Osteomyelitis Post-Operative in a 9 Year Old Child. MEDICAL SCIENCE 24: 2482-86.

R. Tasheva. Physiotherapy after surgically stabilized proximal tibia fracture. Trakia Journal of Sciences. 2020; 18(2):156-160.

Reddy, S., and N. Dhaniwala. 2019 Diaphyseal Fractures in Pediatric Age Group in Rural Area: A Demographic Study." Journal of Datta Meghe Institute of Medical Sciences University 14: 189-91. https://doi.org/10.4103/ jdmimsu.jdmimsu_79_19.

Samal, Nitin, Sanjay Deshpande, Vasant Gawande, and Romil Rati. 2014 Minimal Invasive Percutaneous Plate Osteosynthesis for Distal Tibial Fractures: A Prospective Study. Journal Of Krishna Institute Of Medical Sciences University 3: 120-24.

Shrivastava, Sandeep, Shishir Nawghare, Rajesh Dulani, Pradeep Singh, and Shudhatma Jain. 2009 A Rare Variant of Tibial Hemimelia and Its Treatment. JOURNAL OF PEDIATRIC ORTHOPAEDICS-PART B 18:220-24.

Vos, Theo, Stephen S Lim, Cristiana Abbafati, Kaja M Abbas, Mohammad Abbasi, Mitra Abbasifard, Mohsen Abbasi-Kangevari, et al. 2019 Global Burden of 369 Diseases and Injuries in 204 Countries and Territories, 1990-2019: A Systematic Analysis for the Global Burden of Disease Study. The Lancet 396:1204-22. https://doi.org/10.1016/S0140-6736(20)30925-9. 